\title{
Alkyl Effects on the Chain Initiation Efficiency of Olefin Polymerization by Cationic Half-sandwich Scandium Catalysts: A DFT Study
}

Xiaohui Kang, ${ }^{a, c}$ Guangli Zhou, ${ }^{a}$ Xingbao Wang, ${ }^{a}$ Jingping Qu, ${ }^{a}$ Zhaomin Hou, ${ }^{a, b, *}$ Yi Luo ${ }^{a, *}$

${ }^{a}$ State Key Laboratory of Fine Chemicals, School of Pharmaceutical Science and Technology, Dalian University of Technology, Dalian 116024, China

${ }^{b}$ Organometallic Chemistry Laboratory and Center for Sustainable Resource Science, RIKEN, 2-1 Hirosawa, Wako, Saitama 351-0198, Japan

${ }^{c}$ CAS Key Lab of Separation Sciences for Analytical Chemistry, National Chromatographic R\&A Center, Dalian Institute of Chemical Physics, Chinese Academy of Sciences (CAS), Dalian, 116023, China.

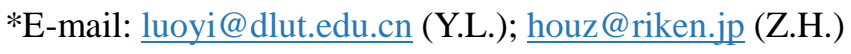

Table S1. Frontier molecular orbital energies and chemical hardness of cationic species 1, 2, and $\mathbf{3}$.

\begin{tabular}{cccc}
\hline \multirow{2}{*}{ Species } & HOMO energy $(\mathrm{eV})$ & LUMO energy $(\mathrm{eV})$ & $\begin{array}{c}\text { Chemical hardness } \\
(\mathrm{eV})\end{array}$ \\
\hline $\mathbf{1}$ & -9.632 & -5.769 & 3.035 \\
$\mathbf{2}$ & -9.142 & -5.197 & 2.999 \\
$\mathbf{3}$ & -9.959 & -6.095 & 3.113 \\
\hline
\end{tabular}

Table S2. HOMO and LUMO energies of ethylene (e), propene (p), 1-hexene (h), styrene (s), trans-butadiene (b), cis-butadiene ( $\left.\mathbf{b}^{\prime}\right)$, trans-isoprene (i), and cis-isoprene (i').

\begin{tabular}{ccc}
\hline Olefin & HOMO $(\mathrm{eV})$ & LUMO $(\mathrm{eV})$ \\
\hline $\mathbf{e}$ & -7.619 & -0.190 \\
$\mathbf{p}$ & -7.102 & 0.136 \\
$\mathbf{h}$ & -7.075 & 0.109 \\
$\mathbf{s}$ & -6.394 & -1.306 \\
$\mathbf{b}$ & -6.585 & -1.116 \\
$\mathbf{b}^{\prime}$ & -6.666 & -1.143 \\
$\mathbf{i}$ & -6.476 & -1.034 \\
$\mathbf{i}^{\prime}$ & -6.530 & -0.929 \\
\hline
\end{tabular}


Table S3. Computed relative free energies of the stationary points involved in the chain initiation of propylene polymerization catalyzed by species $\mathbf{1}, \mathbf{2}$, and $\mathbf{3}$. Energies are relative to $\mathbf{1 / 2 / 3}$ and propylene.

\begin{tabular}{|c|c|c|c|c|}
\hline \multirow{2}{*}{ Species } & \multirow{2}{*}{ Insertion modes } & \multicolumn{3}{|c|}{$\Delta G(\mathrm{kcal} / \mathrm{mol})$} \\
\hline & & $\mathbf{C}$ & TS & $\mathbf{P}$ \\
\hline \multirow{4}{*}{1} & $1,2-r e$ & $-2.8\left(\mathbf{C}^{\mathbf{1}} \mathbf{1 2 p}\right)$ & $7.7\left(\mathbf{T S}^{\mathbf{1}} \mathbf{1 2 p}\right)$ & $-4.4\left(\mathbf{P}^{1}{ }_{12 p}\right)$ \\
\hline & $1,2-s i$ & $-2.8\left(\mathbf{C}^{\mathbf{1}} \mathbf{1 2}^{\prime}\right)$ & $7.7\left(\mathbf{T S}^{1} \mathbf{1 2 p}^{\prime}\right)$ & $-4.4\left(\mathbf{P}^{1} \mathbf{1 2}^{\prime}\right)$ \\
\hline & $2,1-r e$ & $-2.6\left(\mathbf{C}^{\mathbf{1}}{ }_{\mathbf{2 1 p}}\right)$ & $9.8\left(\mathbf{T S}_{\mathbf{2 1}}^{\mathbf{1}}\right)$ & $-2.9\left(\mathbf{P}_{\mathbf{2 1}}^{\mathbf{2 1}}\right)$ \\
\hline & $2,1-s i$ & $-2.6\left(\mathbf{C}^{\mathbf{1}} \mathbf{2 1}^{\prime}\right)$ & $9.8\left(\mathbf{T S}^{\mathbf{1}} \mathbf{2 1}^{\prime}\right)$ & $-2.9\left(\mathbf{P}^{1} \mathbf{2 1}^{\prime}\right)$ \\
\hline \multirow{4}{*}{2} & $1,2-r e$ & $3.0\left(\mathbf{C}^{\mathbf{2}} \mathbf{1 2 p}\right)$ & $19.1\left(\mathbf{T S}^{\mathbf{2}} \mathbf{1 2 p}\right)$ & $3.8\left(\mathbf{P}^{\mathbf{2}} \mathbf{1 2 p}\right)$ \\
\hline & $1,2-s i$ & $3.1\left(\mathbf{C}^{\mathbf{2}} \mathbf{1 2} \mathbf{p}^{\prime}\right)$ & $21.6\left(\mathbf{T S}^{\mathbf{2}} \mathbf{1 2}^{\prime}\right)$ & $4.3\left(\mathbf{P}^{\mathbf{2}} \mathbf{1 2} \mathbf{p}^{\prime}\right)$ \\
\hline & $2,1-r e$ & $4.6\left(\mathbf{C}^{2} \mathbf{2 1 p}\right)$ & $22.0\left(\mathbf{T S}^{2} \mathbf{2 1 p}\right)$ & $9.9\left(\mathbf{P}^{\mathbf{2}} \mathbf{2 1} \mathbf{p}\right)$ \\
\hline & $2,1-s i$ & $3.1\left(\mathbf{C}^{\mathbf{2}} \mathbf{2 1 \mathbf { p } ^ { \prime }}\right)$ & $28.5\left(\mathbf{T S}^{2} \mathbf{2 1}^{\prime}\right)$ & $5.0\left(\mathbf{P}^{2}{ }_{21 \mathbf{p}^{\prime}}\right)$ \\
\hline \multirow{4}{*}{3} & $1,2-r e$ & $-7.7\left(\mathbf{C}^{\mathbf{3}} \mathbf{1 2 p}\right)$ & $7.9\left(\mathbf{T S}^{\mathbf{3}} \mathbf{1 2 p}\right)$ & $-1.0\left(\mathbf{P}^{\mathbf{3}} \mathbf{1 2 p}\right)$ \\
\hline & $1,2-s i$ & $-7.6\left(\mathbf{C}^{3} \mathbf{1 2}^{\prime}\right)$ & $8.1\left(\mathbf{T S}^{\mathbf{3}} \mathbf{1 2}^{\prime}\right)$ & $-4.3\left(\mathbf{P}^{\mathbf{3}} \mathbf{1 2}^{\prime}\right)$ \\
\hline & $2,1-r e$ & $-9.1\left(\mathbf{C}^{\mathbf{3}} \mathbf{2 1}\right)$ & $11.7\left(\mathbf{T S}^{\mathbf{3}} \mathbf{2 1}\right)$ & $-2.6\left(\mathbf{P}^{\mathbf{3}} \mathbf{2 1 p}\right)$ \\
\hline & $2,1-s i$ & $-8.2\left(\mathbf{C}^{\mathbf{3}} \mathbf{2 1} \mathbf{p}^{\prime}\right)$ & $19.8\left(\mathbf{T S}^{\mathbf{3}} \mathbf{2 1 \mathbf { p } ^ { \prime }}\right)$ & $0.6\left(\mathbf{P}^{3} \mathbf{2 1}_{\mathbf{p}^{\prime}}\right)$ \\
\hline
\end{tabular}

Table S4. Computed relative free energies of the stationary points involved in the chain initiation of 1-hexene polymerization catalyzed by species1, 2, and 3. Energies are relative to 1/2/3 and 1-hexene.

\begin{tabular}{|c|c|c|c|c|}
\hline \multirow{2}{*}{ Species } & \multirow{2}{*}{ Insertion modes } & \multicolumn{3}{|c|}{$\Delta G(\mathrm{kcal} / \mathrm{mol})$} \\
\hline & & $\mathbf{C}$ & TS & $\mathbf{P}$ \\
\hline \multirow{4}{*}{1} & $1,2-r e$ & $-4.7\left(\mathbf{C}^{\mathbf{1}} \mathbf{1 2 h}\right)$ & $7.6\left(\mathbf{T S}^{\mathbf{1}} \mathbf{1 2 h}\right)$ & $-3.8\left(\mathbf{P}^{\mathbf{1}} \mathbf{1 2 h}\right)$ \\
\hline & $1,2-s i$ & $-4.7\left(\mathbf{C}^{\mathbf{1}} \mathbf{1 2}^{\prime}\right)$ & $7.6\left(\mathbf{T S}^{\mathbf{1}} \mathbf{1 2}^{\prime}\right)$ & $-3.8\left(\mathbf{P}^{\mathbf{1}} \mathbf{1 2}^{\prime}\right)$ \\
\hline & $2,1-r e$ & $-4.3\left(\mathbf{C}^{\mathbf{1}} \mathbf{2 1 h}\right)$ & $8.5\left(\mathbf{T S}_{\mathbf{2 1 h}}\right)$ & $-2.8\left(\mathbf{P}_{\mathbf{2 1 h}}\right)$ \\
\hline & $2,1-s i$ & $-4.0\left(\mathbf{C}^{\mathbf{1}} \mathbf{2 1}^{\prime}\right)$ & $9.2\left(\mathbf{T S}^{\mathbf{1}} \mathbf{2 1}^{\prime}\right)$ & $-1.9\left(\mathbf{P}^{\mathbf{1}} \mathbf{2 1}^{\prime}\right)$ \\
\hline \multirow{4}{*}{2} & 1,2-re & $2.8\left(\mathbf{C}^{\mathbf{2}} \mathbf{1 2 h}\right)$ & $18.9\left(\mathbf{T S}^{\mathbf{2}} \mathbf{1 2 h}\right)$ & $4.6\left(\mathbf{P}^{\mathbf{2}} \mathbf{1 2 h}\right)$ \\
\hline & $1,2-s i$ & $2.6\left(\mathbf{C}^{\mathbf{2}} \mathbf{1 2 \mathbf { h } ^ { \prime }}\right)$ & $23.2\left(\mathbf{T S}^{\mathbf{2}} \mathbf{1 2 h}^{\prime}\right)$ & $4.8\left(\mathbf{P}^{\mathbf{2}} \mathbf{1 2 h}^{\prime}\right)$ \\
\hline & $2,1-r e$ & $3.1\left(\mathbf{C}^{2} \mathbf{2 1 h}\right)$ & $22.6\left(\mathbf{T S}^{2}{ }^{21 h}\right)$ & $12.2\left(\mathbf{P}^{2} \mathbf{2 1 h}\right)$ \\
\hline & $2,1-s i$ & $2.2\left(\mathbf{C}^{2} \mathbf{2 1 h}^{\prime}\right)$ & $27.1\left(\mathbf{T S}^{\mathbf{2}} \mathbf{2 1 h}^{\prime}\right)$ & $5.3\left(\mathbf{P}^{\mathbf{2}}{ }_{\mathbf{2 1 h ^ { \prime }}}\right)$ \\
\hline \multirow{4}{*}{3} & $1,2-r e$ & $-9.6\left(\mathbf{C}^{\mathbf{3}} \mathbf{1 2 h}\right)$ & $7.3\left(\mathbf{T S}^{\mathbf{3}} \mathbf{1 2 h}\right)$ & $-2.5\left(\mathbf{P}^{\mathbf{3}_{12 h}}\right)$ \\
\hline & $1,2-s i$ & $-9.2\left(\mathbf{C}^{\mathbf{3}} \mathbf{1 2 h}^{\prime}\right)$ & $7.2\left(\mathbf{T S}^{\mathbf{3}} \mathbf{1 2 h}^{\prime}\right)$ & $-2.9\left(\mathbf{P}^{\mathbf{3}} \mathbf{1 2 h}^{\prime}\right)$ \\
\hline & $2,1-r e$ & $-10.4\left(\mathbf{C}^{\mathbf{3}}{ }_{\mathbf{2 1 h}}\right)$ & $12.7\left(\mathbf{T S}^{\mathbf{3}}{ }_{\mathbf{2 1 h}}\right)$ & $-3.8\left(\mathbf{P}^{\mathbf{3}_{\mathbf{2 1 h}}}\right)$ \\
\hline & $2,1-s i$ & $-10.2\left(\mathbf{C}^{\mathbf{3}} \mathbf{2 1}^{\prime}\right)$ & $19.3\left(\mathbf{T S}^{\mathbf{3}} \mathbf{2 1}^{\prime}\right)$ & $-0.3\left(\mathbf{P}^{\mathbf{3}} \mathbf{2 1}^{\prime}\right)$ \\
\hline
\end{tabular}




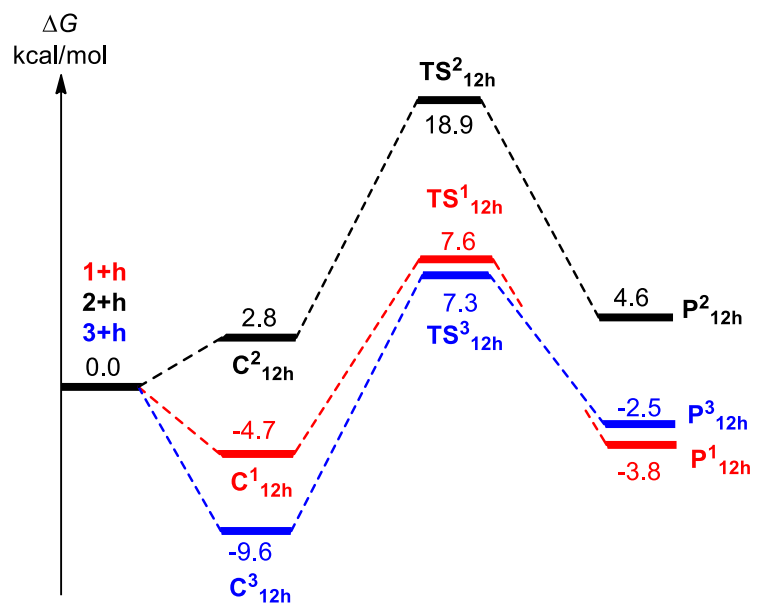

Figure S1. Computed free energy profiles for the chain initiation of 1-hexene polymerization catalyzed by cationic species $\mathbf{1}, \mathbf{2}$, and $\mathbf{3}$. Free energies are relative to $\mathbf{1 / 2} / \mathbf{3}$ and 1-hexene.

Table S5. Computed relative free energies of the stationary points involved in the chain initiation of styrene polymerization catalyzed by species 1, 2, and 3 . Energies are relative to 1/2/3 and styrene.

\begin{tabular}{|c|c|c|c|c|}
\hline \multirow{2}{*}{ Species } & \multirow{2}{*}{ Insertion modes } & \multicolumn{3}{|c|}{$\Delta G(\mathrm{kcal} / \mathrm{mol})$} \\
\hline & & $\mathbf{C}$ & TS & $\mathbf{P}$ \\
\hline \multirow{4}{*}{1} & $1,2-s i$ & $-4.5\left(\mathbf{C}^{\mathbf{1}}{ }^{12 s^{\prime}}\right)$ & $10.8\left(\mathbf{T S}^{\mathbf{1}} \mathbf{1 2 s}^{\prime}\right)$ & $-0.6\left(\mathbf{P}^{\mathbf{1}}{ }_{12 s^{\prime}}\right)$ \\
\hline & $1,2-r e$ & $-4.7\left(\mathbf{C}^{\mathbf{1}} \mathbf{1 2 s}\right)$ & $11.1\left(\mathbf{T S}^{\mathbf{1}}{ }_{12 \mathrm{~s}}\right)$ & $1.4\left(\mathbf{P}^{\mathbf{1}_{12}}\right)$ \\
\hline & $2,1-s i$ & $-0.6\left(\mathbf{C}^{\mathbf{1}}{ }_{21 s^{\prime}}\right)$ & $5.5\left(\mathbf{T S}^{\mathbf{1}}{ }_{21 \mathbf{s}^{\prime}}\right)$ & $-10.1\left(\mathbf{P}^{1} \mathbf{2 1 s}^{\prime}\right)$ \\
\hline & $2,1-r e$ & $-4.6\left(\mathbf{C}^{\mathbf{1}}{ }_{21 \mathrm{~s}}\right)$ & $14.7\left(\mathbf{T S}^{\mathbf{1}} \mathbf{2 1 s}_{\mathbf{s}}\right)$ & $-2.2\left(\mathbf{P}^{\mathbf{1}}{ }_{\mathbf{2 1 s}}\right)$ \\
\hline \multirow{4}{*}{2} & $1,2-s i$ & $2.6\left(\mathbf{C}^{2} \mathbf{1 2 s}^{\prime}\right)$ & $22.5\left(\mathbf{T S}^{2}{ }_{12 \mathbf{s}^{\prime}}\right)$ & $6.9\left(\mathbf{P}^{2}{ }_{12 s^{\prime}}\right)$ \\
\hline & $1,2-r e$ & $1.5\left(\mathbf{C}^{2}{ }_{12 \mathrm{~s}}\right)$ & $24.3\left(\mathbf{T S}^{\mathbf{2}} \mathbf{1 2 s}\right)$ & $8.0\left(\mathbf{P}^{\mathbf{2}} \mathbf{1 2}\right)$ \\
\hline & $2,1-s i$ & $1.7\left(\mathbf{C}^{2}{ }_{21 s^{\prime}}\right)$ & $22.3\left(\mathbf{T S}^{\mathbf{2}}{ }_{21 \mathbf{s}^{\prime}}\right)$ & $9.3\left(\mathbf{P}^{2} \mathbf{2 1}^{\prime}\right)$ \\
\hline & $2,1-r e$ & $15\left(\mathbf{C}^{2} 21 s\right)$ & $28.5\left(\mathbf{T S}^{2}{ }^{21 s}\right)$ & $10.6\left(\mathbf{P}^{2}{ }_{21 s}\right)$ \\
\hline \multirow{4}{*}{3} & $1,2-s i$ & $-9.2\left(\mathbf{C}^{\mathbf{3}} \mathbf{1 2}^{\prime}\right)$ & $16.1\left(\mathbf{T S}^{\mathbf{3}_{12 \mathbf{s}^{\prime}}}\right)$ & $1.2\left(\mathbf{P}^{\mathbf{3}} \mathbf{1 2}^{\prime}\right)$ \\
\hline & $1,2-r e$ & $-11.4\left(\mathbf{C}^{\mathbf{3}} \mathbf{1 2 s}\right)$ & $12.0\left(\mathbf{T S}^{\mathbf{3}}{ }_{12 \mathrm{~s}}\right)$ & $-7.2\left(\mathbf{P}^{\mathbf{3}}{ }_{12 \mathrm{~s}}\right)$ \\
\hline & $2,1-s i$ & $-10.9\left(\mathbf{C}^{\mathbf{3}} \mathbf{2 1}^{\prime}\right)$ & $4.9\left(\mathbf{T S}^{\mathbf{3}}{ }_{\mathbf{2 1 s ^ { \prime }}}\right)$ & $-11.9\left(\mathbf{P}^{\mathbf{3}}{ }_{21 \mathbf{s}^{\prime}}\right)$ \\
\hline & $2,1-r e$ & $-9.2\left(\mathbf{C}^{\mathbf{3}} \mathbf{2 1 s}\right)$ & $15.2\left(\mathbf{T S}^{\mathbf{3}} \mathbf{2 1 s}\right)$ & $-6.8\left(\mathbf{P}^{3}{ }_{21 s}\right)$ \\
\hline
\end{tabular}


Table S6. Computed relative free energies of the stationary points involved in the chain initiation of butadiene polymerization catalyzed by species $\mathbf{1}, \mathbf{2}$, and $\mathbf{3}$. Energies are relative to $\mathbf{1 / 2 / 3}$ and trans-butadiene. The symbol "/" means the failure to locate the stationary points.

\begin{tabular}{|c|c|c|c|c|}
\hline \multirow{2}{*}{ Species } & \multirow{2}{*}{ Insertion modes } & \multicolumn{3}{|c|}{$\Delta G(\mathrm{kcal} / \mathrm{mol})$} \\
\hline & & $\mathbf{C}$ & TS & $\mathbf{P}$ \\
\hline \multirow{8}{*}{1} & trans-1,2-si & -3.7 & 8.9 & -0.4 \\
\hline & trans-1,2-re & -3.7 & 12.1 & 1.0 \\
\hline & trans-1,4-supine & $-2.2\left(\mathbf{C}^{\mathbf{1}} \mathbf{1 4 b}\right)$ & $4.6\left(\mathbf{T S}^{\mathbf{1}} \mathbf{1 4 b}\right)$ & $-19.6\left(\mathbf{P}^{\mathbf{1}}{ }_{\mathbf{1 4 b}}\right)$ \\
\hline & trans-1,4-prone & -3.9 & 13.1 & -17.4 \\
\hline & $c i s-1,2-s i$ & -1.1 & 11.4 & 1.1 \\
\hline & cis-1,2-re & -0.4 & 12.4 & 1.0 \\
\hline & cis-1,4-supine & 1.7 & 13.9 & -18.3 \\
\hline & cis-1,4-prone & $-1.6\left(\mathbf{C}^{\mathbf{1}} \mathbf{1 4}_{\mathbf{b}^{\prime}}\right)$ & $2.4\left(\mathbf{T S}^{\mathbf{1}} \mathbf{1 4}^{\prime}\right)$ & $-20.1\left(\mathbf{P}^{\mathbf{1}} \mathbf{1 4}^{\prime}\right)$ \\
\hline \multirow{8}{*}{2} & trans-1,2-si & 3.6 & 23.2 & 7.9 \\
\hline & trans-1,2-re & 2.7 & 24.3 & 8.7 \\
\hline & trans-1,4-supine & $6.5\left(\mathbf{C}^{2} \mathbf{1 4 b}\right)$ & $19.2\left(\mathbf{T S}^{\mathbf{2}} \mathbf{1 4 b}\right)$ & $-5.2\left(\mathbf{P}^{\mathbf{2}} \mathbf{1 4 b}\right)$ \\
\hline & trans-1,4-prone & l & I & l \\
\hline & $c i s-1,2-s i$ & 6.6 & 23.9 & 9.3 \\
\hline & cis-1,2-re & 6.0 & 25.0 & 9.7 \\
\hline & cis-1,4-supine & 7.2 & 28.7 & 3.3 \\
\hline & cis-1,4-prone & $6.0\left(\mathbf{C}^{\mathbf{2}} \mathbf{1 4 \mathbf { b } ^ { \prime }}\right)$ & $17.4\left(\mathbf{T S}^{\mathbf{2}} \mathbf{1 4}^{\prime}\right)$ & $-7.0\left(\mathbf{P}^{\mathbf{2}} \mathbf{1 4 \mathbf { b } ^ { \prime }}\right)$ \\
\hline \multirow{8}{*}{3} & trans-1,2-si & -8.8 & 17.3 & 1.8 \\
\hline & trans-1,2-re & -8.8 & 16.1 & -5.9 \\
\hline & trans-1,4-supine & $-8.8\left(\mathbf{C}^{\mathbf{3}} \mathbf{1 4 b}\right)$ & $6.76\left(\mathbf{T S}^{\mathbf{3}_{14 b}}\right)$ & $-21.66\left(\mathbf{P}^{\mathbf{3}_{14 b}}\right)$ \\
\hline & trans-1,4-prone & -8.8 & 11.1 & -20.9 \\
\hline & cis-1,2-si & -5.5 & 15.8 & -8.5 \\
\hline & cis-1,2-re & -0.5 & 12.5 & -7.8 \\
\hline & cis-1,4-supine & -5.5 & 6.5 & -18.2 \\
\hline & cis-1,4-prone & $-4.3\left(\mathbf{C}^{\mathbf{3}} \mathbf{1 4 \mathbf { b } ^ { \prime }}\right)$ & $0.2\left(\mathbf{T S}^{\mathbf{3}} \mathbf{1 4}^{\prime}\right)$ & $-21.2\left(\mathbf{P}^{\mathbf{3}} \mathbf{1 4}_{\mathbf{b}^{\prime}}\right)$ \\
\hline
\end{tabular}


Table S7. Computed relative free energies of the stationary points involved in the chain initiation of isoprene polymerization catalyzed by species $\mathbf{1}, \mathbf{2}$, and $\mathbf{3}$. Energies are relative to $\mathbf{1 / 2 / 3}$ and trans-isoprene. The symbol "/" means the failure to locate the stationary points.

\begin{tabular}{|c|c|c|c|c|}
\hline \multirow{2}{*}{ species } & \multirow{2}{*}{ Insertion modes } & \multicolumn{3}{|c|}{$\Delta G(\mathrm{kcal} / \mathrm{mol})$} \\
\hline & & $\mathbf{C}$ & TS & $\mathbf{P}$ \\
\hline \multirow{8}{*}{1} & trans-4,3-si & -3.9 & 12.1 & 2.1 \\
\hline & trans-1,2-re & -3.5 & 13.6 & 4.2 \\
\hline & trans-1,4-supine & $-1.5\left(\mathbf{C}^{\mathbf{1}} \mathbf{1 4 i}\right)$ & $5.8\left(\mathbf{T S}^{\mathbf{1}}{ }_{\mathbf{1 4 i}}\right)$ & $-20.2\left(\mathbf{P}^{1}{ }_{14 \mathbf{i}}\right)$ \\
\hline & trans-1,4-prone & -5.2 & 16.2 & -18.0 \\
\hline & $c i s-4,3-s i$ & -0.7 & 13.2 & 2.01 \\
\hline & cis-4,3-re & -0.8 & 14.0 & -2.2 \\
\hline & cis-1,4-supine & 0.5 & 12.1 & -20.2 \\
\hline & cis-1,4-prone & $-1.2\left(\mathbf{C}^{\mathbf{1}} \mathbf{1 4 i}^{\prime}\right)$ & $3.7\left(\mathbf{T S}^{\mathbf{1}} \mathbf{1 4 i}^{\prime}\right)$ & $-19.1\left(\mathbf{P}^{\mathbf{1}}{ }_{\mathbf{1 4}} \mathbf{i}^{\prime}\right)$ \\
\hline \multirow{8}{*}{2} & trans-4,3-si & 3.8 & 24.0 & 8.8 \\
\hline & trans-4,3-re & 3.4 & 25.1 & 8.6 \\
\hline & trans-1,4-supine & $7.6\left(\mathbf{C}^{\mathbf{2}} \mathbf{1 4 i}\right)$ & $21.0\left(\mathbf{T S}^{\mathbf{2}} \mathbf{1 4 i}\right)$ & $-2.3\left(\mathbf{P}^{\mathbf{2}} \mathbf{1 4 i}\right)$ \\
\hline & trans-1,4-prone & l & l & l \\
\hline & $c i s-4,3-s i$ & 6.5 & 24.3 & 9.3 \\
\hline & cis-4,3-re & 5.5 & 26.4 & 9.6 \\
\hline & cis-1,4-supine & 5.3 & 27.4 & 2.9 \\
\hline & cis-1,4-prone & $8.0\left(\mathbf{C}^{\mathbf{2}}{ }^{14 \mathbf{i}^{\prime}}\right)$ & $20.4\left(\mathbf{T S}^{\mathbf{2}} \mathbf{1 4}^{\prime}\right)$ & $-3.4\left(\mathbf{P}^{\mathbf{2}}{ }^{14 \mathbf{i}^{\prime}}\right)$ \\
\hline \multirow{8}{*}{3} & trans-4,3-si & -9.6 & 17.4 & 2.3 \\
\hline & trans-4,3-re & -9.9 & 11.9 & 1.9 \\
\hline & trans-1,4-supine & $-8.8\left(\mathbf{C}^{\mathbf{3}} \mathbf{1 4 i}\right)$ & $7.7\left(\mathbf{T S}^{\mathbf{3}} \mathbf{1 4 i}\right)$ & $-20.6\left(\mathbf{P}^{\mathbf{3}_{14 i}}\right)$ \\
\hline & trans-1,4-prone & -5.5 & 13.0 & -20.8 \\
\hline & $c i s-4,3-s i$ & -8.3 & 15.2 & -7.7 \\
\hline & cis-4,3-re & -8.3 & 11.3 & -8.5 \\
\hline & cis-1,4-supine & -8.3 & 4.5 & -19.4 \\
\hline & cis-1,4-prone & $-4.4\left(\mathbf{C}^{\mathbf{3}} \mathbf{1 4 i ^ { \prime }}\right)$ & $1.9\left(\mathbf{T S}^{\mathbf{3}_{\mathbf{1 4 i}}}{ }^{\prime}\right)$ & $-19.6\left(\mathbf{P}^{\mathbf{3}_{14 i^{\prime}}}\right)$ \\
\hline
\end{tabular}




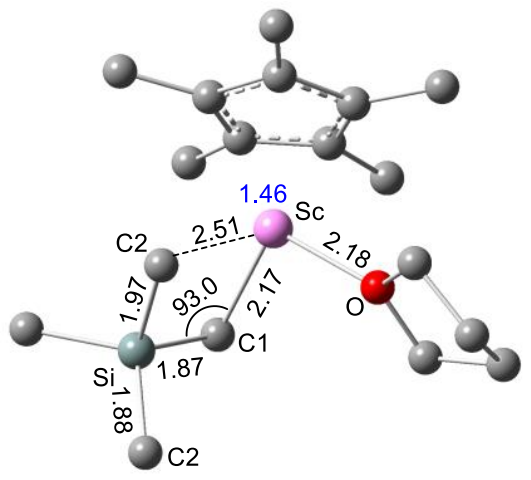

Figure S2. Geometric structures (distances in $\AA$ and angles in degree) of cationic half-sandwich scandium alkyl species $\mathbf{1}^{\prime}$.

Table S8. Computed relative free energies of the stationary points involved in the chain initiations of olefin polymerization by species $\mathbf{1}^{\prime}$. Energies are relative to the energy sum of $\mathbf{1}^{\prime}$ and related monomer.

\begin{tabular}{cccc}
\hline Monomers & \multicolumn{2}{c}{$\Delta G(\mathrm{kcal} / \mathrm{mol})$} & \\
\hline ethylene & $\mathbf{C}$ & $\mathbf{T S}$ & -7.4 \\
\hline propylene & 8.2 & 15.4 & 1.2 \\
\hline 1-hexene & 8.2 & 20.9 & 1.4 \\
\hline styrene & 8.5 & 19.6 & -7.6 \\
\hline cis-butadiene & 6.1 & 17.5 & -13.3 \\
\hline cis-isoprene & 15.7 & 18.0 & -10.1 \\
\hline
\end{tabular}

